\title{
Rashba effect in InGaAs/InP parallel quantum wires
}

\author{
V. A. Guzenko, J. Knobbe, H. Hardtdegen, and Th. Schäpers ${ }^{a)}$ \\ Institute of Thin Films and Interfaces (ISG 1) and Virtual Institute of Spin Electronics (VISel), Research \\ Centre Jülich GmbH, 52425 Jülich, Germany \\ A. Bringer \\ Institute of Solid State Research (IFF) and CNI-Center of Nanoelectronic Systems for Information \\ Technology, Research Centre Jülich GmbH, 52425 Jülich, Germany
}

(Received 29 September 2005; accepted 15 December 2005; published online 17 January 2006)

\begin{abstract}
We report on the Rashba effect in InGaAs/InP quantum wires with an effective width ranging from $1.18 \mu \mathrm{m}$ down to $210 \mathrm{~nm}$. By measuring 160 wires in parallel universal conductance, fluctuations could be suppressed so that the characteristic beating effect in the magnetorestistance was observable down to very low magnetic fields. A characteristic shift of the nodes in the beating pattern was found for decreasing wire width. By assuming a realistic soft-wall potential, the experimentally observed node positions could be reproduced. For the range of measured wires, our study confirms that the Rashba coupling parameter does not change with wire width. (C) 2006 American Institute of Physics. [DOI: 10.1063/1.2165279]
\end{abstract}

The concept of many recently proposed spin electronic devices relies on one-dimensional semiconductor structures in conjunction with the Rashba effect. Here, the basic idea is the control of the spin orientation by utilizing the Rashba spin-orbit coupling. ${ }^{1}$ In these structures, the inversion asymmetry imposed by an asymmetric macroscopic potential profile of a semiconductor quantum well results in lifting of the spin degeneracy. The Rashba spin-orbit coupling leads to a spin precession of propagating electrons. By means of a gate electrode on top of the quantum well, the shape of the potential profile and thus the degree of spin-precession can be controlled. ${ }^{2,3}$ This mechanism opens up the possibility to design electronic devices, where the switching process is realized by changing the spin orientation in the semiconductor. Indeed, this scheme is the basis for the well-known spin transistor proposed by by Datta and Das. ${ }^{4}$ Very recently, a number of spin electronic devices have been proposed, which explicitly make use of one-dimensional channels, e.g., nonmagnetic spin-filters, ${ }^{5}$ spin-polarizers, ${ }^{6}$ or ring-shaped interference devices. ${ }^{7}$

In order to realize one of the structures discussed above, it is essential to gain detailed knowledge about the transport properties of one-dimensional structures with Rashba spinorbit coupling. Theoretically their transport properties have been investigated first by Moroz and Barnes ${ }^{8}$ and by Mireles and Kirczenow. ${ }^{9}$ Regarding experimental studies on wire structures, the presence of Rashba spin-orbit coupling was confirmed by observing a characteristic beating pattern in the magnetoresistance, ${ }^{10,11}$ similar to the case of a twodimensional electron gas (2DEG). ${ }^{12,13}$ However, for very narrow wires a systematic shift of the node of the beating pattern was found. Qualitatively, this shift could be explained by the effect of the geometrical confinement on the magnetosubbands in the semiconductor, as was theoretically shown for a wire with parabolic confinement potential. ${ }^{14}$

In this letter, we report on a concise experimental and theoretical investigation of the Rashba effect in InGaAs/InP quantum wires. In contrast to previous experiments, a large

${ }^{a)}$ Electronic mail: th.schaepers@fz-juelich.de number of wires have been measured in parallel. This allowed us to suppress universal conductance fluctuations, which are usually superimposed on the Shubnikov-de Haas $(\mathrm{SdH})$ oscillations. Consequently, the beating pattern in the magnetoresistance could be resolved down to very low magnetic fields. A systematic change of the beating pattern was found for narrower wires. In order to explain the measured results, detailed simulations of the energy spectrum were performed taking a realistic potential profile into account.

The $\mathrm{In}_{0.53} \mathrm{Ga}_{0.47} \mathrm{As} / \mathrm{In}_{0.77} \mathrm{Ga}_{0.23} \mathrm{As} / \mathrm{InP}$ heterostructure used for our study was grown by metalorganic vapor phase epitaxy on a semi-insulating InP substrate. The 2DEG is located in a strained $10 \mathrm{~nm}$ thick $\operatorname{In}_{0.77} \mathrm{Ga}_{0.23}$ As layer. The lower barrier of the quantum well is formed by an InP layer, while for the upper layer a $70 \mathrm{~nm}$ thick $\mathrm{In}_{0.53} \mathrm{Ga}_{0.47} \mathrm{As}$ layer is used. The electrons are provided by a $10 \mathrm{~nm}$ thick InP dopant layer separated by $20 \mathrm{~nm}$ of InP from the quantum well. Electron-beam lithography and reactive ion etching were employed to define the quantum wire structures. ${ }^{11} \mathrm{~A}$ layer sequence of $\mathrm{Ni} / \mathrm{AuGe} / \mathrm{Ni} / \mathrm{Au}$ was used to prepare ohmic contacts. A number of 160 identical wires each $620 \mu \mathrm{m}$ long were connected in parallel. Five sets of wires were investigated with a geometrical width ranging from $1220 \mathrm{~nm}$ down to $340 \mathrm{~nm}$. From the analysis of the $\mathrm{SdH}$ oscillations of a Hall bar sample (Fig. 1), a carrier concentration of $n_{2 \mathrm{D}}=5.3 \times 10^{11} \mathrm{~cm}^{-2}$ and a mobility of $\mu$ $=205000 \mathrm{~cm}^{2} / \mathrm{V} \mathrm{s}$ at $0.6 \mathrm{~K}$ were determined. A clear beating pattern was observed in the $\mathrm{SdH}$ oscillations indicating the presence of Rashba spin-orbit coupling. For the 2DEG a Rashba coupling parameter of $\alpha_{R}=4.84 \times 10^{-12} \mathrm{eV}$ m was extracted from the $1 / B$ fast Fourier transform. ${ }^{15}$

As can be seen in Fig. 1(a), a pronounced beating pattern is observed in the magnetoresistance $R_{x x}$ for all sets of quantum wires, except for the set of very narrow wires $(340 \mathrm{~nm})$. From the oscillations at higher magnetic fields, it was confirmed that the electron concentration of all wires corresponds to the concentration of the 2DEG. For the following analysis of the transport properties, the relevant width of the wires is the effective electrical width $W_{\text {eff }}$. Information on this parameter can be obtained from the position $B_{\max }$ of the 

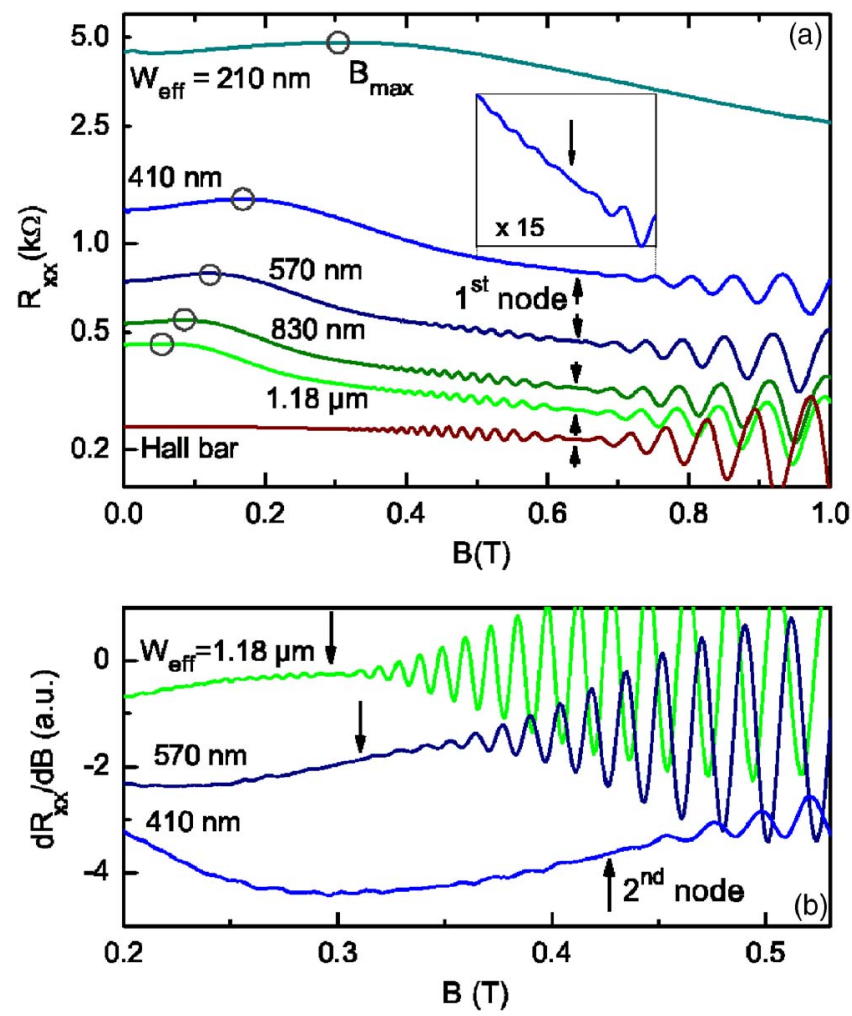

FIG. 1. (Color online) (a) SdH oscillations of quantum wires of different effective widths (log-scale) at $0.6 \mathrm{~K}$. The first-order node of the beating pattern is indicated by an arrow. The position of $B_{\max }$ is indicated by a circle. The inset shows a magnified signal around the first-order node of the $410 \mathrm{~nm}$ wide wire. (b) $d R_{x x} / d B$ vs $B$ of the $1.18 \mu \mathrm{m}, 570 \mathrm{~nm}$, and $410 \mathrm{~nm}$ wide wires. The second-order nodes are indicated by arrows.

broad resistance maximum observed at low magnetic fields [marked by a circle in Fig. 1(a)]: $W_{\text {eff }} \approx 0.55 k_{F} / e B_{\max } \cdot{ }^{16,17}$ Here, $k_{F}$ is the Fermi wave number. The peak at $B_{\max }$ originates from diffusive boundary scattering. Owing to carrier depletion at the boundary of the quantum wires, the effective width is smaller than the geometrical width, i.e., $W_{\text {eff }}$ of the narrowest set of wires is $210 \mathrm{~nm}$ compared to the geometrical width of $340 \mathrm{~nm}$. For the following, we will exclusively refer to the effective width.

A closer look on the beating pattern of the different sets of wires [Fig. 1(a)] reveals that the position of the first-order node does not change with decreasing wire width. In contrast, the second-order node initially located at $0.3 \mathrm{~T}$ for the Hall bar structure shifts to higher magnetic fields for narrower wires. In order to better identify the position of the second-order node, the differential resistance $d R_{x x} / d B$ was calculated [Fig. 1(b)]. The magnetoresistance of the narrowest wires $\left(W_{\text {eff }}=210 \mathrm{~nm}\right)$ does not show any oscillations below $1 \mathrm{~T}$ due to the strong effect of diffusive boundary scattering. The node positions of the beating patterns for all wire widths can be found in Fig. 2.

The beating pattern in the magnetoresistance results from an interplay of an external magnetic field $B$ and spinorbit interaction. Without spin-orbit coupling, the magnetic field organizes the spectrum of the electrons in a 2DEG in a set of degenerate Landau levels. Their energies increase linearly with $B$. The spin-orbit interaction mixes the Landau levels and leads to an inhomogeneous level density, responsible for the beating pattern. For a 2DEG, the energy levels can be determined by an analytical formula for the magne-

Downloaded 21 Dec 2006 to 134.94.122.39. Redistribution subject

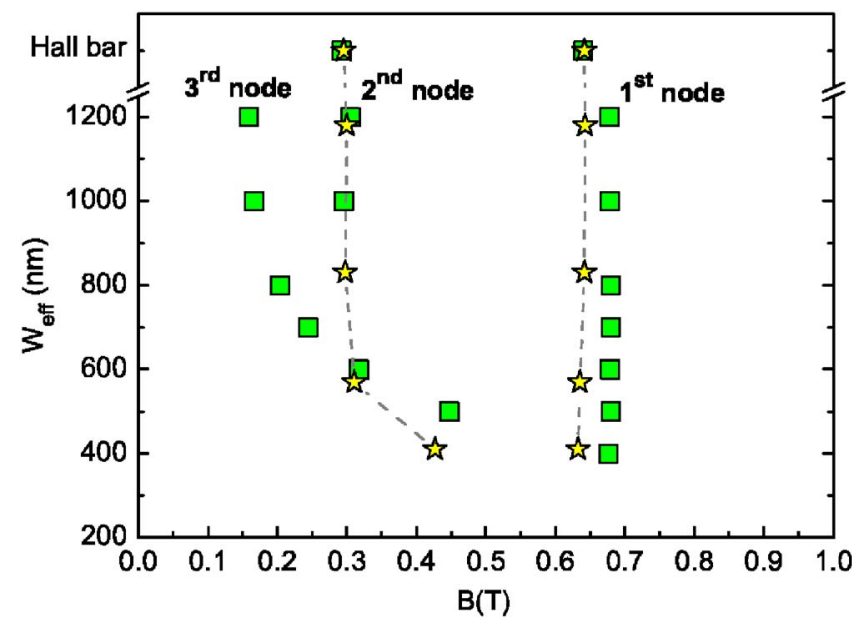

FIG. 2. (Color online) Positions of the first- and second-order nodes of the beating pattern experimentally observed in the magnetoresistance $(\star)$. The node positions obtained from the simulation are marked by filled squares.

toresistance depending on $B, \alpha_{R}$, the Fermi energy $E_{F}$, the effective mass $m^{*}$, the $g$ factor of the electron spins, and the level broadening $\Gamma$, due to impurity scattering. ${ }^{2,13}$ The material parameters $m^{*}, g, E_{F}, \alpha_{R}$, and $\Gamma$ for our 2DEG had been determined by a least mean-square fit of the theoretical formula to the experimentally measured magnetoresistance [see Fig. 3(a)]. We find for our sample: $m^{*}=0.039 m_{e}$, $g=-4, E_{F}=32.74 \mathrm{meV}$, and $\Gamma=0.74 \mathrm{meV}$. For the Rashba coupling parameter, a value of $\alpha_{R}=5.38 \times 10^{-12} \mathrm{eV} \mathrm{m}$ was found. The fact that the experimentally obtained value of $\alpha_{R}$ is smaller can be attributed to the inaccuracy connected to the extraction of $\alpha_{R}$ by a fast Fourier transform. ${ }^{15}$

With increasing $B$, the Landau levels cross the Fermi energy. Spin-orbit coupling strongly affects the rate by which this crossing takes place. This allows a direct determination of the node position in the $\mathrm{SdH}$ oscillations, as illustrated in Fig. 3. When plotting the $B$ positions at which the energy of a Landau level equals $E_{F}$ versus their spacing, one finds positions, marked by $M$, with neighboring levels almost degenerate and positions $N$ with equal spacing between levels. As

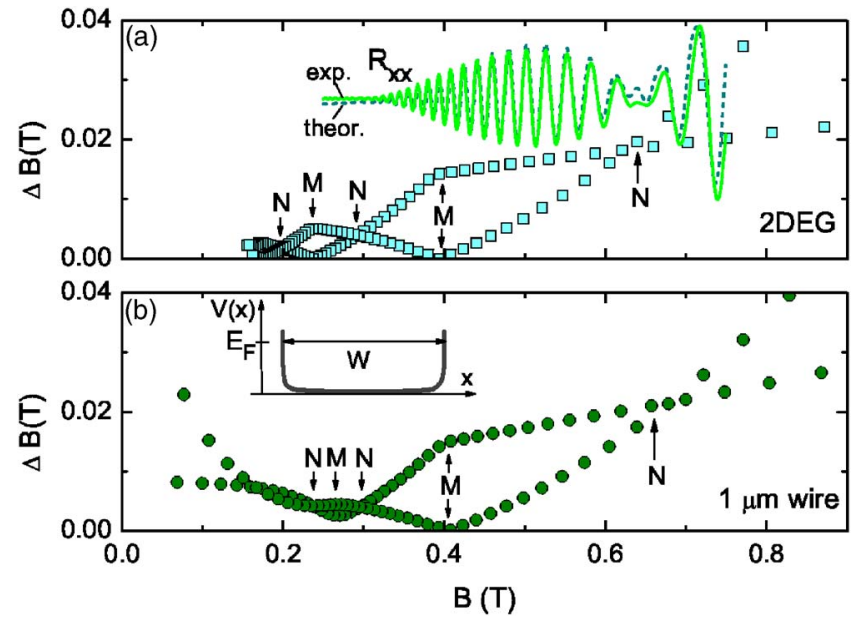

FIG. 3. (Color online) Spacing of neighboring Landau levels crossing $E_{F}$ at different magnetic fields for a 2DEG (a) and a $1 \mu \mathrm{m}$ wide wire (b). Position of nodes $(\mathrm{N})$ and maxima $(\mathrm{M})$ in the beating pattern of SdH oscillations. The experimental and theoretical curve of $R_{x x}$ of the 2DEG is added in (a) for comparison. A schematic illustration of the wire potential is shown in (b) as an inset. 
can be seen by direct comparison to the experimental curve shown in Fig. 3(a), at $N$ the $\mathrm{SdH}$ oscillations have a node, while at $M$ a large amplitude is found.

For wires of limited width $W$, no analytical solution for the electron levels is known in presence of spin-orbit coupling and a magnetic field, but there is a numerical solution for a system laterally confined by a parabolic potential. The parabolic confinement is normally chosen to discuss the properties of quantum wires. However, as shown by simulations of quasi one-dimensional systems with $W$ much larger than the Fermi wavelength, it is more appropriate to model the electron confinement by a quasi-rectangular soft-wallike potential, as sketched in Fig. 3(b) (inset). ${ }^{18}$ We therefore developed an algorithm to solve the Schrödinger equation for electrons in a wire for an arbitrary form of the confining potential. With this algorithm, we were able to calculate the $B$ positions with level energies equal $E_{F}$.

The spectrum for electrons in a wire transforms gradually from degenerate Landau levels to quasi one-dimensional subbands, when the diameter of the Landau orbit gets comparable to $W$ with decreasing $B$. When $W$ decreases more and more, nodes in the beating pattern disappear and below a critical $W$ there is no node anymore. For our structure with an assumed soft-wall potential, this critical width is roughly $400 \mathrm{~nm}$, as can be seen in Fig. 2. Up to $600 \mathrm{~nm}$, two nodes can be detected. Between $600 \mathrm{~nm}$ and $800 \mathrm{~nm}$, the second node splits so that for $W>600 \mathrm{~nm}$ a third additional node can clearly be identified.

We determined the nodal positions with the material parameters found for the 2DEG and they agree well with the experimentally observed positions, as can be seen in Fig. 2. However, the third-order node expected from the simulation could not be resolved experimentally, due to level broadening by scattering processes. The first node remains at approximately the same position for all wire widths, since at these large magnetic fields the Landau energy exceeds the confinement energy. For parabolic confinement, the positions of the nodes depend on $W$ much more strongly so that no reasonable agreement with experiment could be achieved. ${ }^{14}$ Furthermore, variation of $\alpha_{R}$ in the simulation led to a large deviation of the nodes from the experimentally observed ones. Thus, our simulations confirm that $\alpha_{R}$ does not change with $W$, at least down to the wire width where no beating pattern could be observed anymore.

In conclusion, by measuring parallel InGaAs/InP quantum wires, the beating effect in the $\mathrm{SdH}$ oscillations could be resolved down to very low magnetic fields. The shift of the nodes in the beating pattern with decreasing wire width could be explained by the additional quantization due to carrier confinement in a soft-wall potential.

${ }^{1}$ E. I. Rashba, Fiz. Tverd. Tela (Leningrad) 2, 1224 (1960) [Sov. Phys. Solid State 2, 1109 (1960)].

${ }^{2}$ J. Nitta, T. Akazaki, H. Takayanagi, and T. Enoki, Phys. Rev. Lett. 78, 1335 (1997)

${ }^{3}$ G. Engels, J. Lange, Th. Schäpers, and H. Lüth, Phys. Rev. B 55, R1958 (1997).

${ }^{4}$ S. Datta and B. Das, Appl. Phys. Lett. 56, 665 (1990).

${ }^{5}$ A. A. Kiselev and K. W. Kim, Appl. Phys. Lett. 78, 775 (2001).

${ }^{6} \mathrm{M}$. Governale, D. Boese, U. Zülicke, and C. Schroll, Phys. Rev. B 65, 140403 (2002).

${ }^{7}$ J. Nitta, F. E. Meijer, and H. Takayanagi, Appl. Phys. Lett. 75, 695 (1999).

${ }^{8}$ A. V. Moroz and C. H. W. Barnes, Phys. Rev. B 61, R2464 (2000).

${ }^{9}$ F. Mireles and G. Kirczenow, Phys. Rev. B 64, 024426 (2001).

${ }^{10}$ Y. Sato, S. Gozu, T. Kikutani, and S. Yamada, Physica E (Amsterdam) 272, 114 (1999).

${ }^{11}$ Th. Schäpers, J. Knobbe, and V. A. Guzenko, Phys. Rev. B 69, 235323 (2004).

${ }^{12}$ J. Luo, H. Munekata, F. F. Fang, and P. J. Stiles, Phys. Rev. B 38, 10142 (1988).

${ }^{13}$ B. Das, D. C. Miller, S. Datta, R. Reifenberger, W. P. Hong, P. K. Bhattacharya, J. Singh, and M. Jaffe, Phys. Rev. B 39, 1411 (1989).

${ }^{14}$ J. Knobbe and Th. Schäpers, Phys. Rev. B 71, 035311 (2005).

${ }^{15}$ Th. Schäpers, G. Engels, J. Lange, T. Klocke, M. Hollfelder, and H. Lüth, J. Appl. Phys. 83, 4324 (1998).

${ }^{16}$ T. J. Thornton, M. L. Roukes, A. Scherer, and B. P. V. de Gaag, Phys. Rev. Lett. 63, 2128 (1989).

${ }^{17}$ S. Block, M. Suhrke, S. Wilke, A. Menschig, H. Schweizer, and D. Grützmacher, Phys. Rev. B 47, 6524 (1993).

${ }^{18}$ S. E. Laux, D. J. Frank, and F. Stern, Surf. Sci. 196, 101 (1988). 